\title{
Entertainment Computing in the Orbit
}

\author{
Matthias Rauterberg ${ }^{1}$, Mark Neerincx ${ }^{2}$, Karl Tuyls ${ }^{1}$, Jack van Loon $^{3}$
}

\author{
${ }^{1}$ Eindhoven University of Technology, The Netherlands \\ ${ }^{2}$ TNO Human Factors \& TU Delft, The Netherlands \\ ${ }^{3}$ DESC Space Flight/Microgravity \& ACTA Free University Amsterdam, The Netherlands \\ g.w.m.rauterberg@tue.nl, mark.neerincx@tno.nl, j.vanloon@vumc.nl
}

\begin{abstract}
During ultra long space missions (i.e. to Mars), the isolated space environment affects a number of physiological, psychosocial and mental processes critically involved in human performance, and it is vital to missions' success to understand the psychological limits. Past experiences in space have shown that the mental health of a crew can have a great effect on the success or failure of a mission. Latent and overt stress factors are mental strain, interpersonal problems, and lack of capability to rescue crew members, isolation, monotony, and tedium of life aboard an autonomous shuttle. Abstract These issues develop very slowly over time and are very difficult to detect and remedy for observers on the ground. E.g. long-term isolation can lead to sleep deprivation, depression, irritability, anxiety, impaired cognition, and even hostility. Providing astronauts with entertainment products can help to maintain the mental health of the crew. The results of this project will deepen the understanding of intra- and inter-individual crew behaviour and related performance, and provide the technical platform for a new type of crew assistance tools based on multi-user computer games.
\end{abstract} Keywords: astronaut, space research, entertainment, game, mental health.

\section{Introduction}

The European Space Agency (ESA) has started to roadmap the necessary research for making a manned mission to Mars feasible (see Fig. 1). In 2005 the TU Eindhoven was contacted by the Dutch Experiment Support Center (DESC) to start collaboration on research in entertainment and mental health of a crew on ultralong space flights (e.g. to Mars). As one of the outcomes of this discussion was setting-up the Dutch Entertainment Computing Consortium (DECC) ${ }^{1}$. In 2006

\footnotetext{
${ }^{1}$ See http://www.org.id.tue.nl/DECC/
} 
DECC organized a workshop at the Lorentz Center (Leiden, The Netherlands) ${ }^{2}$ to establish discussions among Dutch researchers in the field of entertainment and health, training and serious gaming. This research project brought together the available state of the art expertise in the Netherlands on man-machine interaction and entertainment, human factors and cognitive task load, evolutionary game theory and multi-agent systems, and Actor-Agent Community technology at DECIS Lab (Delft).

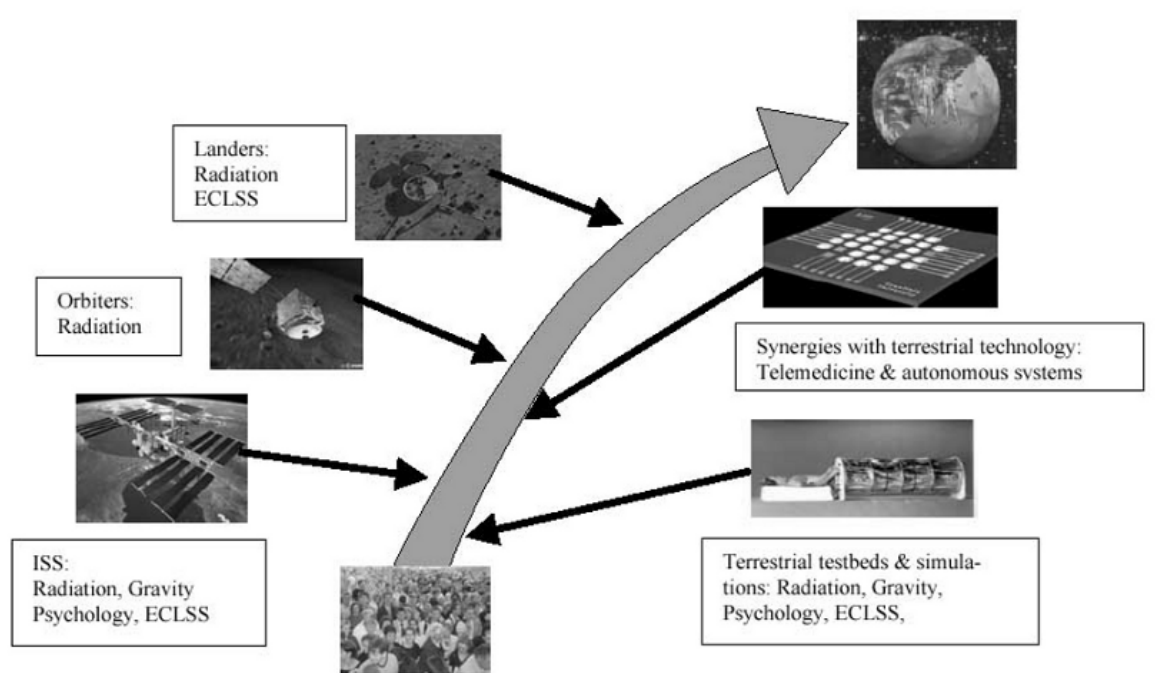

Fig 1: Roadmap with regard to human health issues for a future European strategy towards human exploratory missions.

\section{State of the art}

The success of human space missions depends among others on the following two factors: (1) each astronaut remains alert and vigilant while operating sophisticated equipment and following complex procedures, and (2) the whole crew can operate and collaborate autonomously and successfully to recover from critical situations. Ultra-long space missions are characterized on the one hand by new physical parameters, on the other hand by new psychosocial parameters. New physical parameters are weightlessness, three-dimensional configuration, and reduced living

\footnotetext{
${ }^{2}$ See http://www.lorentzcenter.n1/lc/web/2006/214/info.php3?wsid=214
} 
space. New psychosocial parameters are small group living, working together in close proximity, and extreme social isolation (Manzey, 2004). Adaptation to this new environment involves a number of mental processes, which includes simultaneously physiological, psychosocial and behavioural modifications in the course of time (Christensen and Talbot, 1986; Tafforin, 1996; Palinkas, 2001; Sandal, 2001a; Manzey, 2004). It is reported that the Russian scientists have taken definitely more interest in studying psychosocial factors affecting their cosmonauts than the American (Kanas, 1985, 1987; Santy, 1983). In spite of a very careful selection and preparation of the crew members, including a test of "psychological compatibility", within a long-term mission already after 30 days the crew began to show signs of hostility (Kanas, 1987).

The space environment in ultra-long missions affects a number of physiological systems critically involved in human performance, and it is vital to mission success to understand the biological, physiological and psychological limits of human performance under such conditions (Palinkas, 2001). It has been demonstrated that both acute gravitational changes and isolation in ultra-long space flight (e.g., social monotony) can lead to stress and performance problems (Endler, 2004). Since e.g. isolation and hyper-arousal result in both psychosocial and performance deficits, this project is focused on (1) a diagnostic tool to measure psychosocial crew health status, and (2) countermeasures to assist crew members improving team communication and collaboration (i.e. [semi] Autonomous Mental Health Assistance, AMHA; see also Rauterberg, 2004). In particular, this project is concerned with the following aspects: altered work-leisure time cycles with related mood problems, and altered or reduced communication and collaboration opportunities among crew members with related possible inter-individual conflicts.

\section{Risks and Goals:}

In the human behaviour and performance discipline area the following three risks have been identified as unacceptable risks of serious adverse health and performance consequences without having so far a mitigation strategy that has been demonstrated on Earth or even validated in space (ESTEC 2001; NASA 2004):

- Risk 1--Area: Human Behaviour and Performance

Risk Title: Human performance failure due to poor psychosocial adaptation.

Risk Description: Human performance failure due to problems associated with adapting interpersonally to the space environment: poor interpersonal relationships and/or group dynamics; and inadequate team cohesiveness.

Justification: Moderate likelihood/high consequence risk with low mitigation status; need to reduce probability of human error, performance and/or mission failure. Serious interpersonal conflicts have occurred in space flights. The failure of flight crews to cooperate and work effectively with each other or with flight controllers has been a periodic problem in both US and Russian space flight programs. Interpersonal distrust, dislike, misunderstanding and poor communi- 
cation have led to potential dangerous situations, such as crew members refusing to speak to one another during critical operations, or withdrawing from voice communication with ground controllers. Such problems of group cohesiveness have a high likelihood of occurrence in ultra-long space flight and if not mitigated through prevention or intervention, they will pose grave risks to the mission.

[References: Kanas 1985, 1987; Palinkas 1991, 2001; Suedfeld and Steel 2000; Morphew 2001; Sandal 2001a, 2001b]

Countermeasures: Unobtrusive monitoring and early detection of individual adaptation problems.

- Risk 2--Area: Human Behaviour and Performance.

Risk Title: Human performance failure due to neurobehavioral problems.

Risk Description: Human performance failure during missions due to such conditions as depression, anxiety, trauma, or other neuro-psychiatric and cognitive problems.

Justification: Although infrequent, serious neurobehavioral problems involving stress and depression have occurred in space flight, especially during longduration missions. In some of these instances, the distress has contributed to performance errors during critical operations, such as the collision of Progress into Mir during manual docking. In other instances, emotional problems led to changes in motivation, diet, sleep, and exercise. No matter how carefully selected and prepared crews are for long-duration flights, the US and Russian experiences reveal that at least some subset of astronauts will experience problems with their behavioural health. Long-duration flights will have a significant likelihood of psychiatric problems emerging.

[References: Santy 1987; Kanas 1998; Driskell et al. 1999; Ellis 2000; Palinkas and Houseal 2000; Suedfeld and Steel 2000; Lane and Feeback 2002; Woolford et al. 2002; Kanas 2004; Palinkas et al. 2004]

Countermeasures: On-board unobtrusive technologies as astronaut's aids for valid detection of stress reactions and cognitive or emotional problems; on-board information technologies as astronauts' aids for management of stress reactions and cognitive or emotional problems; self monitoring of mood; improved diagnostic cognitive self-assessment; improved ability to safely and effectively manage an uncooperative crewmember during mission.

- Risk 3--Area: Space Human Factors Engineering.

Risk Title: Mismatch between crew cognitive capabilities and task demands.

Risk Description: Human performance failure due to inadequate accommodation of human cognitive limitations and capabilities. If human cognitive performance capabilities are surpassed due to inadequate design of tools, interfaces, tasks or information support systems, mission failure or decreased effectiveness or efficiency may result. Identifying, locating, processing, or evaluating information to make decisions and perform critical tasks in short time-frames in nominal and emergency situations, with limited crew size, relying on strictly lo- 
cal resources is extremely subject to human errors.

Justification: Crew members require refresher training and information support systems for numerous tasks during six or more month missions. Psychological literature documents increases in error with time since learning and decreases in error with correctly practicing task. Failure to correctly follow procedures has lead to fatal accidents in commercial aviation, even with greatly over learned tasks.

[References: Torrance 1954; Tafforin 1996; Ellis 2000; Woolford et al. 2002; Endler 2004]

Countermeasures: Tools for enabling crew autonomy with respect to information retrieval; tools analyzing tasks to identify critical skills and knowledge; tools to enable self-assessment of readiness to perform; onboard training systems that enables successful readiness to perform. Design requirements for communications systems among crewmembers, between crew and mission control, and among crew and intelligent agents that reduce risk of mission failure.

The scope and size of this project does not allow covering the whole area of all three risks, but we propose an innovative countermeasure that addresses several issues in each of these risks. The proposed [semi] AMHA is a desktop virtual reality software that provides a new type of computer mediated communication and collaboration space among crew members, between each crew member and his/her personal buddy/coach in form of a persuasive virtual agent (De Haan et al. 2005). Based on empirical research, diagnostic relevant characteristics of commercially available desktop virtual reality applications will be extracted, and the results will be implemented into the new AMHA. The virtual agent (buddy) will be adaptive in its communication behaviour to serve the following two main purposes: (1) establishing a trustworthy and private relationship to the particular crew member (Neerincx and Streefkerk 2003), and (2) providing an indirect communication channel to other 'buddies' and crew members (Neerincx et al. 2006).

\section{Scientific objectives}

The scientific objectives are twofold: (1) empirical research investigating communication and collaboration pattern of crews based on objective behavioural patterns, and (2) software engineering research designing and implementing a new type of crew assistance tool using agent technology. The outcome of the empirical research will be used as input for the technical specifications and later for validation of the prototype.

Goal 1: Reduce the risk of human physiological or psychosocial performance failure by investigating countermeasures based on adaptive single- and multi-user game/agent technology for unobtrusive onboard training, counseling and diagnostic of individual and crew behavior. 
Goal 2: Develop new methods and tools for unobtrusive monitoring of actual mental and social status of crew cooperation and individual performance guaranteeing the privacy of crew members.

Goal 3: Develop Earth-based applications of adaptive multi-user game/agent technologies for diagnosing, assessing, and training crew members to reduce the risk of mental and psychosocial performance failure.

In the next section we describe the technical objectives to realize the AMHA. The final result will be a running prototype to demonstrate and validate relevant concepts and functionality, and to investigate and assess the potential of our proposed approach for future research in this direction.

\subsection{Research methodology and technical feasibility}

This project will executed in three phases: (phase 1) data gathering and analysis in the context of a confinement study with commercial available software (i.e., single and multi user applications) added with automatic user actions logging; (phase 2) developing a software platform to demonstrate the basic concepts of the diagnostic functionality and intended computer mediated communication for the AMHA; and (phase 3) empirical validation of the developed AMHA prototype (cf. Neerincx and Lindenberg 2007). Diagnostic relevant and valid information about the actual relationship among crew members has to satisfy the following requirements: (1) unconstrained behavior for starting and ending collaboration, (2) all crew members should be getting involved, and (3) unobtrusive measuring procedures. Any application that can fulfill these basic requirements seems to be a possible platform. Before we describe each phase in more detail we have to introduce some basic concepts of desktop virtual reality worlds in the context of simulation and gaming, and our conclusions related to this project.

A multiuser online role-playing game (MORPG) is an online computer roleplaying game (RPG) in which a number of users interact with one another in a desktop virtual reality world. As in all RPGs, users assume the role of a virtual character and take control over many of that character's actions. MORPGs are distinguished from single-user or small multi-user RPGs by the number of users, and by the game's persistent world, usually hosted by the game's publisher. A persistent world is a desktop virtual reality world that is used as a setting for a roleplaying game, often online. The world is always available and world events happen continually. The persistency comes from maintaining and developing the state of the gaming world around the clock. Unlike with other games, a persistent world game's plots and events continue to develop even while some of the users are not playing their character. The comparison is to the real world where events occur that are not directly connected to a user, or continue to happen while a user sleeps, 
etc. Likewise, a user's character can also influence and change a persistent world. The degree to which a character can affect a world varies from game to game. Persistent worlds do also exist in offline games. Even though technically nothing happens while the game is off, the illusion of persistency is created by advancing events as soon as the game is turned on and using the game engine's clock as a guide for what should have happened, making it seem like events occurred while the game was off. MORPGs are considered by Bonk and Dennen (2005) as serious candidates for assessment and training in the military domain. There are many different user types in a MORPG environment. In a fairly simple taxonomy, Bartle (1996) identified four key roles: achievers, explorers, socializers, and killers. These roles may arise from the interrelationship of two dimensions of playing style: (1) action versus interaction, and (2) world-oriented versus player-oriented. In addition, role assignments have the potential to be an important factor in educational use. For example, it is possible that certain roles foster particular types of learning. Users could be encouraged to assume roles that are most fitting with their task requirements. Alternatively, they might be required to rotate through roles to experience the computer mediated collaboration space from a variety of perspectives.

Conclusion: We propose to build upon such a desktop virtual reality world in form of a MORPG enhanced with virtual agents ('buddies'). Crew members can login at any time to continue their on-going interactions inside this world. In particular they can set up a private relationship with their buddy for communication, and with other crew members for collaborations.

Autonomy is a social notion, and recent research in artificial intelligence has been linked to many social theories. Delegation theory is one such social theory. In many cases the user (or the delegating agent) needs local and decentralized knowledge and decision from the delegated agent (Tuyls and Nowe 2005). This agent-- delegated to take care of a given task-- has to choose from among different possible plans, or to adapt abstract or previous plans to suit new situations; it has to find additional (local and updated) information; it has to solve a problem and not just to execute a function, an action, or implement a recipe; sometimes it has to exploit its 'expertise'. In all these cases this agent takes care of the interests or goals of the former 'remotely', i.e., far from it and without its monitoring and intervention (control), and autonomously. This requires an 'open delegation' (Hexmoor et al. 2008). The virtual agent is supposed to use its knowledge, its intelligence, and its ability, and to exert a degree of discretion.

Conclusion: We propose to design the buddy agent with sufficient autonomy for a set of different tasks (e.g., assessing the mental health status of the related crewmember and providing individual feedback only to protect privacy; communicating with other buddies to assess the entire psychosocial crew status, checking and updating email connection with Earth, etc.).

Social norms are cultural phenomena that naturally emerge in human societies and help prescribe and proscribe normative patterns of behavior. In recent times, the discipline of multi-agent systems has been modeling social norms in artificial 
society of agents. Hexmoor et al. (2006) reviews norms in multi-agent systems and then offers exploration of a series of norms in a simulation study. Using game theoretic concepts they define and offer an account of norm stability. Particularly in small groups, for the norm of cooperation to evolve and be stable, a relatively small number of individuals with cooperative attitude are needed.

Conclusion: We propose to build in such functionality for the virtual agents to enable social norm stability among crewmembers.

Over the last few years the combination of Evolutionary Game Theory (EGT) and Reinforcement Learning (RL) have proved to be powerful theories for designing autonomous agents, and under-standing interactions in systems composed of such agents (Tuyls et al. 2006). Modeling agents for an assistive tool requires a thorough understanding of the type and form of interactions within the virtual environment and other agents in the tool controlled by users. Instead of assuming that agents are perfectly rational, EGT treats agents' objectives as a matter of fact, with a presumption that these objectives must be compatible with an appropriate evolutionary dynamic (i.e., the replicator dynamics). For this reason, EGT can be used to analyze how agents can make less purely self-interested decisions in complex environments such as adaptive games (Spronk 2005).

Conclusion: Modeling and implementing the virtual agents ('buddy') will be based on EGT and RL.

Conventional media technologies have been designed to primarily handle multimedia, informative, logical communications based on logic and aiming for the user's understanding of a message. This is a narrow view on the communication capabilities of humans and Kansei Mediation offers new opportunities (Nakatsu et al. 2006). It is a style of lifelong learning that possesses a rich combination of communication channels to let direct and indirect information flow freely. Nakatsu et al. (2006) discusses how human-human communication can be delivered indirectly using different modalities. The metaphor of the 'four-ears-model' distinguishes four important dimensions of any human message: (1) content ("what are the facts"), (2) appeal ("what does s/he want me to do, think, feel, etc."), (3) relationship ("what does s/he think about me") and (4) self-disclosure ("what kind of person is s/he"). In communicating the sender sends messages about the subject, which tell something about him/herself about his perception of the relationship with the receiver and which appeal to the receiver to change in some way. One can say the sender talks with four different 'beaks'. To understand the sender well the receiver should listen with four 'ears' and each 'ear' should be tuned to what the corresponding 'beak' tries to say. If the 'beak' and the 'ear' are not in tune with each other, this is one of the main causes for misunderstandings.

Conclusion: We propose to enhance the user interface in addition to visual and audio with haptic feedback.

A first informal user requirement analysis was done with the astronaut Gerhard Thiele (ESA-EAC). He recommends the following requirements: (1) mixed gen- 
der crew, (2) strict confidentiality and privacy for the interaction with and through AMHA, and (3) adaptive tools (e.g., e-learning) to support crew-members achieving their private learning goals throughout a mission.

Conclusion: We will design the interaction with AMHA so that adaptation and privacy is guaranteed.

Due to the microgravity constrains under real ultra long flight conditions any interface concept based on tangible interactions seems to be not appropriate (see Rauterberg et al., 1997).

\subsection{Work plan}

For the first phase of this project we plan to get access to a confinement study for long duration missions (e.g., Mars-500 confinement study, ESA space flights to ISS) as an empirical test bed. We plan to provide a PC platform with a set of commercial games: for individual social interaction (e.g., Sims-2), for individual training (e.g. SpaceStationSim, BioSim), and for inter individual interactions (e.g., Age of Empires, Baldur's Gate, XBlast TNT). This PC platform might be extended with a special logging functionality (e.g. NOLDUS uLog Pro plus Observer XT) to collect automatically the objective data generated by users during inter-actions. We will relate the results with additional subjective data gathered by questionnaires and interviews (e.g., a sociogram) to extract diagnostic relevant information out of the logged data stream (Rauterberg 2003). All necessary facilities [e.g. special analyzing tools like Automatic Mental Model Evaluator, AMME (Rauterberg 1993)] are available within our laboratories.

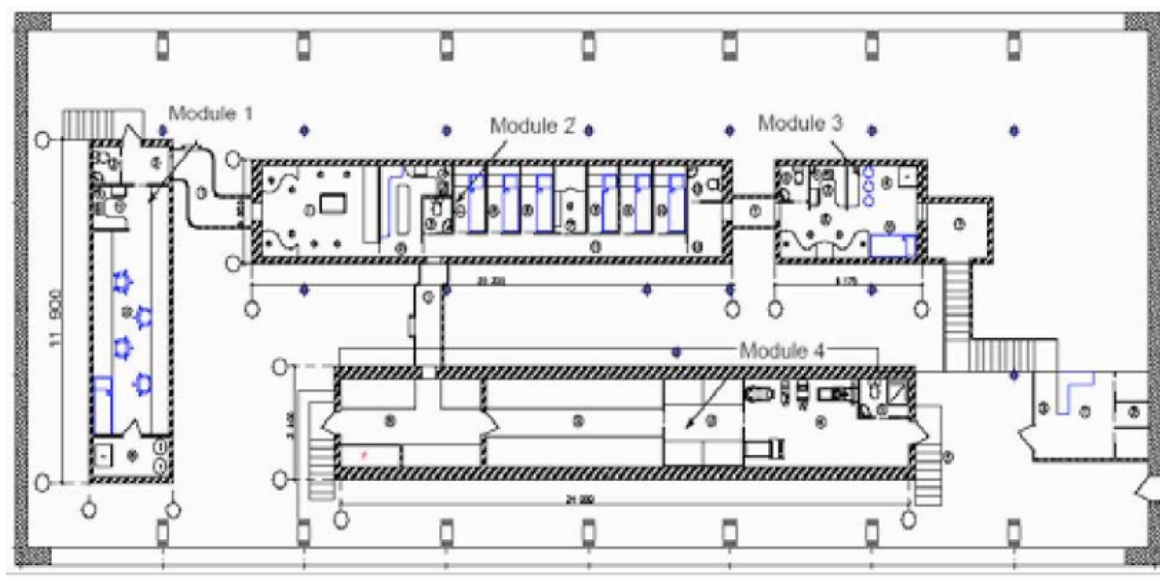

Fig 2: Layout of the mock space station: module 1=medical module, module $2=$ living quarters, module $3=$ Mars landing module, module $4=$ storage module $^{3}$

\footnotetext{
${ }^{3}$ Taken from

http://spaceflight.esa.int/users/downloads/ao2007/Mars500\%20Call\%20for\%20Candidates.pdf
} 


\section{The Mars-500 confinement study}

European and Russian space researchers will lock six men in a metal confinement to mimic the stresses and challenges of a manned mission to Mars. First a 100 and later a 500 days experiment, under development by the Russian Institute of Medical and Biological Problems, will put human volunteers in a mock space station module (see Fig. 2) in complete isolation to study how a ultra-long mission to Mars might affect its human crew (Johnson et al. 2003).

During these two studies, the crew will depend on a preset limit of supplies, including about five tons of food and oxygen and three tons of water. A doctor will accompany volunteers inside the module to treat illnesses and injuries. Volunteers will be allowed to quit the experiment only if they develop a severe ailment of psychological stress. During the simulation the crew will watch Earth disappear into the blackness of space as they munch on the same kinds of food available on the International Space Station. ESA will even attempt to simulate the 40-minute time delay for radio signals to travel from the spacecraft to Earth and back, as the crew work and live in extremely isolated conditions.

\section{Conclusions}

It is obvious that serious research still has to be done to investigate the effects of ultra-long space missions in a confined environment under microgravity constrains on the mental health of the crew members. Any possible countermeasures to minimize these risks are necessary to contribute to missions' success. The proposed project AMHA is one reasonable contribution in this direction.

Acknowledgments. This project is sponsored by the Netherlands Organisation for Scientific Research (NWO), User Support Program Space Research (SRON) and DECIS Lab. We want to thank J. Goldstein, K. Nieuwenhuis, P. Suedfeld and B. Salem for their valuable contributions to our discussions.

\section{References}

Bartle R A (1996). Hearts, clubs, diamonds, spades: Players who suit MUDs. Journal of MUD Research, vol. 1(1). Retrieved November 10, 2006 from http://www.mud.co.uk/richard/hcds.htm.

Bonk C J and Dennen V P (2005). Massive multiplayer online gaming: a research framework for military training and education. ADL Technical Report 2005-1, US State Department of Defense.

Christensen J M and Talbot J M (1986). A review of the psychological aspects of space flight. Aviation, Space, and Environmental Medicine, vol. 57(3), pp. 203-212. 
De Haan G, van der Mast C A P G, Blanson Henkemans O and Neerincx M A (2005). SuperAssist: Personal assistants for cooperative healthcare treatment. In: M Al-Akaidi and L Rothkrantz (eds.), Proceedings Euromedia 2005 (pp. 124-128). Toulouse, France: EUROSIS.

Driskell J E, Salas E and Johnston J (1999). Does stress lead to a loss of team perspective? Group Dynamics: Theory, Research, and Practice, vol. 3(4), pp. 291-302.

Ellis S R (2000) Collision in space: human factors elements of the Mir Progress 234 collision. Ergonomics in Design, pp. 4-9.

Endler N S (2004). The joint effects of person and situation factors on stress in spaceflight. Aviation, Space, and Environmental Medicine, vol. 75(7), pp. C22-C27.

ESTEC (2001). HUMEX: Study on the survivability and adaptation of humans to long-duration interplanetary and planetary environments. HUMEX Technical Note 2 (version 1), ESTEC/Contract No. 14056/99/NL/PA.

Hexmoor H, Castelfranchi C and Falcone R (2008, forthcoming). A prospectus on agent autonomy. In: H Hexmoor and S Brainov (eds.), Agent Trust (in planning stage).

Hexmoor H, Venkata S G and Hayes D (2006). Modelling social norms in multiagent systems. Journal of Experimental and Theoretical Artificial Intelligence, vol. 18(1), pp. 49-71.

Johnson J C, Boster J S and Palinkas L A (2003). Social roles and the evolution of networks in extreme and isolated environments. Journal of Mathematical Sociology, vol. 27, pp. 89-121.

Kanas N (1985). Psychosocial factors affecting simulated and actual space missions. Aviation, Space, and Environmental Medicine, vol. 56, pp. 806-811.

Kanas N (1987). Psychological and interpersonal issues in space. American Journal of Psychiatry, vol. 144(6), pp. 703-709.

Kanas N (1998). Psychiatric issues affecting long-duration space missions. Aviation, Space, and Environmental Medicine, vol. 69, pp. 1211-1216.

Kanas N (2004). Group interactions during Space missions. Aviation, Space, and Environmental Medicine, vol. 75(1), pp. C3-C5.

Lane H W and Feeback D L (2002). Habitability and environmental factors: the future of closedenvironment tests. In: H W Lane, R L Sauer and D L Feeback (eds.) ISOLATION: NASA experiments in closed environment living (pp. 419-432). San Diego, CA: American Astronautical Society.

Manzey D (2004). Human missions to Mars: new psychological challenges and research issues. Acta Astronautica, vol. 55, pp. 781-790.

Morphew M E (2001). Psychological and Human Factors in Long Duration Spaceflight. McGill Journal of Medicine, vol. 6(1), pp. 74-80.

Nakatsu R, Rauterberg M and Salem B (2006). Forms and theories of communication: From multimedia to Kansei mediation. Multimedia Systems, vol. 11(3), pp. 304-312.

NASA (2004). Bioastronautics Critical Path Roadmap (BCPR): an approach to risk reduction and management for human space flight--extending the boundaries. Document JSC 62577 (draft), NASA.

Neerincx M A and Streefkerk J W (2003). Interacting in desktop and mobile context: Emotion, trust and task performance. In: Aarts, E., Collier, R., van Loenen, E. \& de Ruyter, B. (Eds.), Ambient Intelligence: EUSAI 2003. Lecture Notes in Computer Science (pp. 119-132), Springer.

Neerincx M A, Lindenberg J, Smets N, Grant T, Bos A, Olmedo Soler A,. Brauer U and Wolff M (2006). Cognitive engineering for long duration missions: human-machine collaboration on the Moon and Mars. In: Proceedings of 2nd IEEE International Conference on Space Mission Challenges for Information Technology (pp. 40-46), Los Alamitos, California: IEEE.

Palinkas L A (1991). Effects of physical and social environments on the health and well-being of Antarctic winter-over personnel. Environment \& Behavior, vol. 23(6), pp. 782-799. 
Palinkas L A (2001). Psychosocial issues in long-term space flights: overview. Gravitational and Space Biology Bulletin, vol. 14(2), pp. 25-33.

Palinkas L A and Houseal M (2000). Stages of change in mood and behaviour during a winter in Antarctica. Environment and Behavior, vol. 32(1), pp. 128-141.

Palinkas L A, Johnson J C and Boster J S (2004). Social support and depressed mood in isolated and confined environments. Acta Astronautica, vol. 54, pp. 639-647.

Rauterberg, M., Bichsel, M., Meier, M., Fjeld, M. (1997). A gesture based interaction technique of a planning tool for construction and design. In: Proceedings 6th IEEE International Workshop on Robot and Human Communication--RO-MAN'97, (IEEE Catalog Number: 97TH8299, pp. 212-217). Piscataway: IEEE Press.

Rauterberg M (1993). AMME: an Automatic Mental Model Evaluation to analyze user behaviour traced in a finite, discrete state space. Ergonomics, vol. 36(11), pp. 1369-1380.

Rauterberg M (2003). Determinants for collaboration in networked multi-user games. In: R Nakatsu and J Hoshino (eds.), Entertainment Computing--Technologies and Applications (pp. 313-321). IFIP, Kluwer Academic Press.

Rauterberg, M. (2004). Positive effects of entertainment technology on human behaviour. In: R. Jacquart (ed.), Building the Information Society (pp. 51-58). IFIP, Kluwer Academic Press.

Sandal G M (2001a). Psychosocial issues space: future challenges. Gravitational and Space Biology Bulletin, vol. 14(2), pp. 47-54.

Sandal G M (2001b). Crew tension during a space station simulation. Environment and Behavior, vol. 33(1), pp. 134-150.

Santy P (1983). The journey out and in: psychiatry and space exploration. American Journal of Psychiatry, vol. 140, pp. 519-527.

Santy P (1987). Psychiatric components of a Health Maintenance Facility (HMF) on space station. Aviation, Space and Environmental Medicine, vol. 58, pp. 1219-1224.

Spronk P (2005). Adaptive Game AI. PhD Thesis, IKAT-University of Maastricht, Netherlands.

Suedfeld P and Steel G D (2000). The environmental psychology of capsule habitants. Annual Review of Psychology, vol. 51, pp. 227-253.

Tafforin C (1996). Ethological analysis of crew member behaviour: distance, orientations, and postures. Advances in Space Biology and Medicine, vol. 5, pp. 263-281.

Torrance E P (1954). The behaviour of small groups under stress conditions of "survival". American Sociological Review, vol. 19(6), pp. 751-755.

Tuyls K and Nowe A (2005). Evolutionary game theory and multi-agent reinforcement learning. The Knowledge Engineering Review, vol. 20(1), pp. 63-90.

Tuyls K, 't Hoen P J and Vanschoenwinkel B (2006). An evolutionary dynamical analysis of multi-agent learning in iterated games. The Journal of Autonomous Agents and Multi-Agent Systems, vol. 12(1), pp. 115 - 153.

Walther J B (1997). Group and interpersonal effects in international computer-mediated collaboration. Human Communication Research, vol. 23(3), pp. 342-369.

Woolford B, Hudy C, Whitmore M, Berman A, Maida J and Pandya A (2002). In Situ training project: LMLSTP phase III report. In: H W Lane, R L Sauer and D L Feeback (eds.), ISOLATION: NASA experiments in closed environment living (pp. 407-417). American Astronautical Society. 